1. Mukherjee, S., et al. 2008. Pharmacologic targeting of a stem/progenitor population in vivo is associated with enhanced bone regeneration in mice. J. Clin. Invest. 118:491-504.

2. Zangari, M., Yaccoby, S., Cavallo, F., Esseltine, D., and Tricot, G. 2006. Response to bortezomib and activation of osteoblasts in multiple myeloma. Clin. Lymphoma Myeloma. 7:109-114.

3. Terpos, E., Sezer, O., Croucher, P., and Dimopoulos, M.A. 2007. Myeloma bone disease and proteasome inhibition therapies. Blood. 110:1098-1104.

4. Lentzsch, S., Ehrlich, L.A., and Roodman, G.D. 2007 Pathophysiology of multiple myeloma bone disease. Hematol. Oncol. Clin. North Am. 21:1035-1049.

5. Giuliani, N., Rizzoli, V., and Roodman, G.D. 2006 Multiple myeloma bone disease: Pathophysiology of osteoblast inhibition. Blood. 108:3992-3996.

6. Sezer, O., Heider, U., Zavrski, I., Kühne, C.A., and Hofbauer, L.C. 2003. RANK ligand and osteoprotegerin in myeloma bone disease. Blood. 101:2094-2098.

7. Terpos, E., et al. 2003. Soluble receptor activator of nuclear factor kappaB ligand-osteoprotegerin ratio predicts survival in multiple myeloma: proposal for a novel prognostic index. Blood. 102:1064-1069.

8. Terpos, E., et al. 2006. Bortezomib reduces serum dickkopf-1 and receptor activator of nuclear factor$\kappa \mathrm{B}$ ligand concentrations and normalizes indices of bone remodeling in patients with relapsed multiple myeloma. Br. J. Haematol. 135:688-692.

9. Zavrski, I., et al. 2005. Proteasome inhibitors abrogate osteoclast differentiation and osteoclast function. Biochem. Biophys. Res. Commun. 333:200-205.

10. von Metzler, I., et al. 2007. Bortezomib inhibits human osteoclastogenesis. Leukemia. 21:2025-2034.

11. Zhao, M., et al. 2004. Smurf1 inhibits osteoblast differentiation and bone formation in vitro and in vivo. J. Biol. Chem. 279:12854-12859.

12. Zhao, M., Qiao, M., Oyajobi, B.O., Mundy, G.R., and Chen, D. 2003. E3 Ubiquitin ligase smurf1 mediates core-binding factor $\alpha 1 /$ Runx 2 degradation and plays a specific role in osteoblast differentiation. J. Biol. Chem. 278:27939-27944.
13. Garrett, I.R., et al. 2003. Selective inhibitors of the osteoblast proteasome stimulate bone formation in vivo and in vitro. J. Clin. Invest. 111:1771-1782.

14. Munemasa, S., et al. 2007. Immunomodulatory thalidomide analogs do not affect osteoprogenitor differentiation and low concentration of bortezomib promotes it, while both agents suppress osteoclast differentiation [abstract]. Blood. 110:4780.

15. Giuliani, N., et al. 2007. The proteasome inhibitor bortezomib affects osteoblast differentiation in vitro and in vivo in multiple myeloma patients. Blood. 110:334-338.

16. Anderson, G., et al. 2006. Thalidomide derivative CC-4047 inhibits osteoclast formation by downregulation of PU.1. Blood. 107:3098-3105.

17. Jilka, R.L. 2007. Molecular and cellular mechanisms of the anabolic effect of intermittent PTH. Bone. 40:1434-1446.

18. Grey, A., et al. 1999. A role for interleukin-6 in parathyroid hormone-induced bone resorption in vivo. Endocrinology. 140:4683-4690.

\title{
Colitis and cancer: a tale of inflammatory cells and their cytokines
}

\author{
Ezra Burstein1,2 and Eric R. Fearon1,2,3,4 \\ ${ }^{1}$ Department of Internal Medicine, ${ }^{2}$ Molecular Mechanisms of Disease Program, ${ }^{3}$ Department of Human Genetics, and \\ ${ }^{4}$ Department of Pathology, University of Michigan Medical School, Ann Arbor, Michigan, USA.
}

\begin{abstract}
Chronic inflammatory disorders are often associated with an increased cancer risk. A particularly striking example of the chronic inflammationcancer link is seen in inflammatory bowel disease, in which chronic colitis or persistent inflammation in the colon is associated with elevated risk of colorectal cancer. Animal models exploring the mechanisms by which inflammation increases the risk of colon cancer have shown that inflammatory cells, through the effects of the cytokines they produce, have a major role in promoting neoplastic transformation. In this issue of the JCI, Popivanova and colleagues demonstrate that TNF- $\alpha$, through its effects on the immune system, plays a critical role in promoting neoplastic transformation in this setting (see the related article beginning on page 560). Importantly, the study also provides evidence that anti-TNF- $\alpha$ therapies, which are currently in clinical use, may interrupt the process.
\end{abstract}

Inflammatory bowel disease (IBD) affects approximately 1.4 million people in the United States, with an estimated annual cost exceeding \$2 billion (1). IBD mainly consists of two disorders, ulcerative colitis (UC) and Crohn disease (CD). UC is restricted to the colon and/or rectum and always involves a continuous segment of variable length starting from the rectum.

Nonstandard abbreviations used: AOM, azoxymethane; $\mathrm{CAC}$, colitis-associated cancer; $\mathrm{CD}$, Crohn disease; CRC, colorectal cancer; DSS, dextran sulfate sodium; $\mathrm{IBD}$, inflammatory bowel disease; IKK, IкB kinase.

Conflict of interest: The authors have declared that no conflict of interest exists.

Citation for this article: J. Clin. Invest. 118:464-467 (2008). doi:10.1172/JCI34831.
$\mathrm{CD}$ is a more varied disorder, which can affect essentially any segment of the gastrointestinal tract, with a preference for the terminal ileum (2). While UC causes inflammation restricted to the mucosa, CD is associated with granulomatous features and transmural inflammation that can be complicated by intestinal wall fibrosis and stenosis, internal and external fistulas, and intra-abdominal infections. The involvement of the colon and rectum, irrespective of the subtype of IBD, increases the risk for colorectal cancer (CRC), and the risk is more pronounced with early onset of the disease and greater severity and greater extent of the colitis (3). In fact, though patients with colitis-associated cancer (CAC) represent only about $1 \%$ of CRC cases, colitis patients are among those in the population at greatest risk of CRC. In patients with prolonged (>20 years) and extensive colitis involving the entire organ, the risk of CRC approaches $20 \%$. Certain subsets of patients, such as those that have concurrent inflammation in the biliary tract (termed "primary sclerosing cholangitis"), have an even greater lifetime risk of CRC, approaching 50\% (3).

The abnormal inflammatory response observed in IBD is thought to require the interplay between host genetic factors and the intestinal microbiota (4). Indeed, some patients with IBD seem to improve upon antibiotic treatment, and multiple animal models of colitis are ameliorated by the administration of antibiotics or placement of animals in germ-free conditions (4). Recently, the demonstration that a subset of $C D$ patients carries mutations in the nucleotide-binding oligomerization domain-containing 2 (NOD2) gene (5-7), which encodes an intracellular pattern-recognition receptor for bacterial muramyldipeptides $(4,8)$, bolsters the notion that an abnormal balance in the immune response to gut bacteria may be a central and general feature in IBD. 


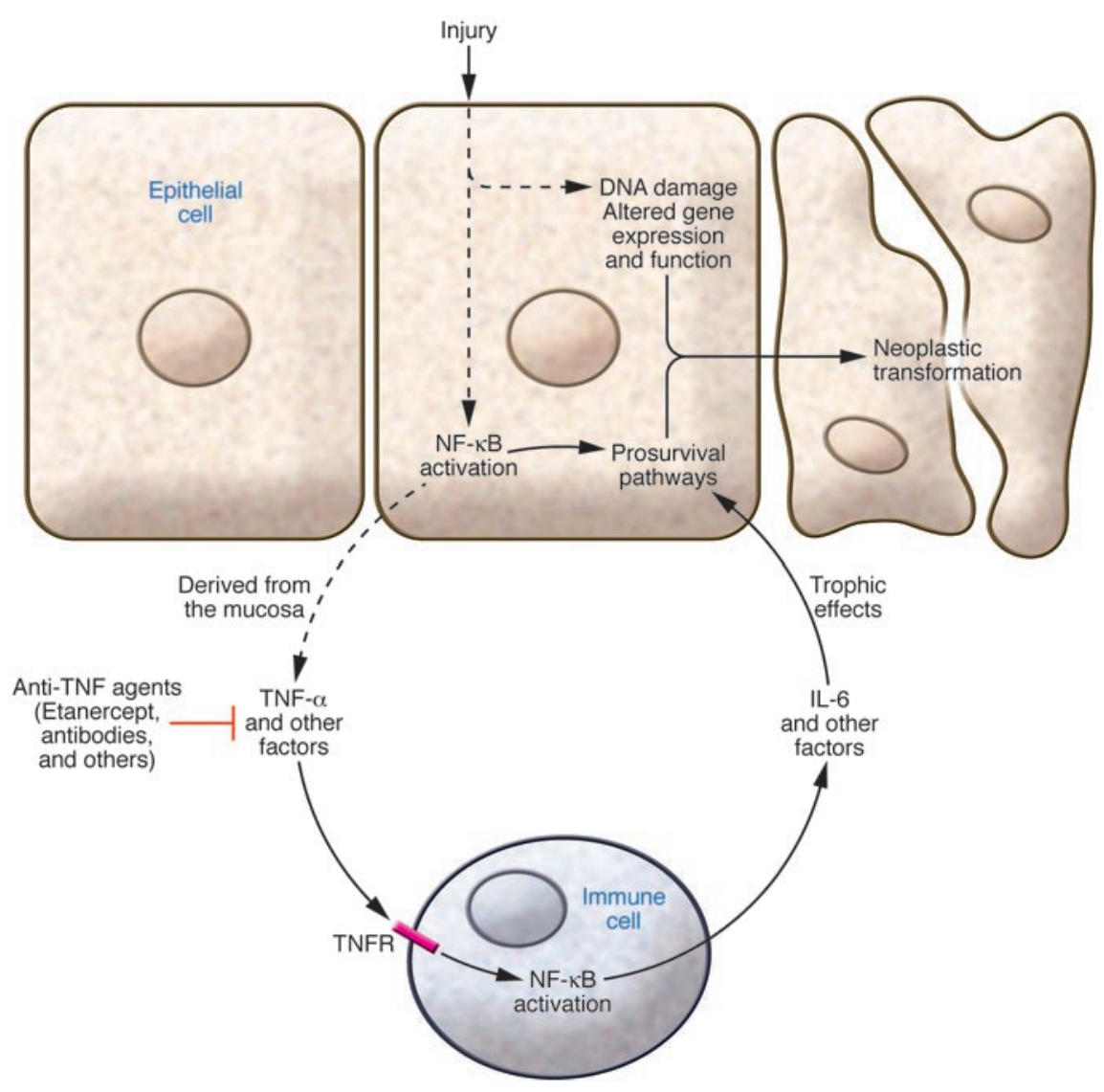

\section{CAC: a paradigm for inflammation- associated cancer}

As noted above, though CAC represents only a small fraction of the overall burden of CRC (3), the molecular pathogenesis of CAC likely represents a paradigm for inflammation-associated carcinogenesis. Adding to the particular interest in CAC has been the development of an animal model that reproducibly leads to colonic neoplasia in the setting of colitis. This model involves the administration of small doses of the carcinogen azoxymethane $(\mathrm{AOM})$, followed by repeated rounds of chemical colitis induced by administration of dextran sulfate sodium (DSS) in the drinking water. By the end of three cycles of colitis, or in about 1-2 months of treatment, nearly $100 \%$ of mice develop neoplasms in the colon (9).

Regardless of the specific inciting events underlying human IBD, a feature that is nearly always observed is the mucosal activation of NF-кB (10), a pleiotropic transcription factor with a key role in innate and adaptive immunity (11). NF- $\mathrm{KB}$ is required for the expression of various proinflammatory factors (11), including mediators that play a critical role in IBD such as cytokines and adhesion molecules $(2,4)$. Indeed, blockade of NF- $\mathrm{KB}$ can ameliorate or prevent the development of colitis in animal models (12-15). In addition to its critical function in inflammation, NF- $\kappa \mathrm{B}$ promotes expression of a number of prosurvival factors and can play an oncogenic role in certain settings, especially in lymphoid malignancies (16). More recently, attention has shifted to the potential role of NF- $\mathrm{BB}$ and mediators that activate NF- $\mathrm{KB}$ in inflammation-associated cancers.

\section{NF-kB: a key player in CAC}

NF- $\kappa \mathrm{B}$ function is regulated by inhibitor

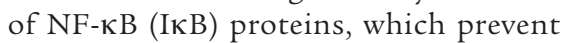
nuclear accumulation and DNA binding

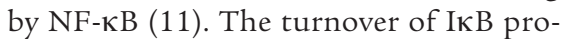
tein is dynamically regulated in response to various stimuli, including proinflammatory cytokines, microbial products, and various forms of cellular stress including DNA damage (11). Ubiquitination and proteasomal degradation of IKB is activated by its phosphorylation, and the kinase responsible is a component of a multimeric complex known as the IкB kinase (IKK) (11). Consistent with the proposed key role of IKK in regulat-

\section{Figure 1}

Role of TNF- $\alpha$ in inflammation associated with colorectal carcinogenesis. Injury to the intestinal epithelium can result in DNA damage and altered gene expression and function, the initial step required for neoplastic transformation. In addition, this is accompanied by activation of NF-KB within epithelial cells, which promotes prosurvival pathways that are required for the initial growth of the resulting neoplastic cells. NF- $\mathrm{KB}$ activation also promotes proinflammatory gene expression. TNF- $\alpha$ originating from the mucosa or possibly the epithelium itself, participates in orchestrating the activation of immune cells. Production of various proinflammatory factors by the activated immune system participates in the ensuing inflammatory response but additionally plays a role in tumor growth by providing trophic signals to the early neoplastic lesions. Loss of TNF- $\alpha$ signaling in immune cells, and not the mucosa, stops this cascade by aborting the mucosal inflammatory response, and this can be achieved by pharmacologic blockade of TNF- $\alpha$ with etanercept and possibly other agents (21). ing $I \kappa B$ and NF- $\kappa B$, genetic inactivation of the IKK $\beta$ subunit in mice abrogates $\mathrm{NF}-\kappa \mathrm{B}$ activation in response to most stimuli (11). Moreover, work from Greten, Karin, and colleagues showed that in the AOM/DSS model tissue-specific inactivation of IKK $\beta$ in the intestinal epithelium dramatically reduced tumor multiplicity, indicating that prosurvival signals provided by NF- $\kappa \mathrm{B}$ probably play a role in colitisassociated tumor initiation (17). On the other hand, this same study reported that deletion of IKK $\beta$ in the myeloid compartment, which greatly impairs most innate immune responses observed, had no significant effect on tumor number but did have a major effect on tumor size (17). This latter observation indicates NF- $\mathrm{KB}$ activation in the immune compartment is important for tumor growth, probably via effects on the expression of certain cytokines, which might in turn have trophic effects on the outgrowth and/or progression of neoplastic colon cancer cells. In fact, modulation of IL- 6 expression has a dramatic effect on tumor growth in experimental models of CAC, suggesting that this cytokine functions as a trophic factor for neoplastic epithelium (18). 
Other pathways that modulate NF-кB activation have been found to also play a role in the AOM/DSS experimental colitis model. Deficiency of the cylindromatosis (CYLD) gene, which encodes a deubiquitinase with an important role in regulating NF-кB activation, results in a tumor-susceptibility syndrome in humans known as cylindromatosis (19). Inactivation of the murine homolog of CYLD enhances CAC formation in the setting of experimental colitis in mice (20), indicating once again that activation of NF- $\mathrm{KB}$ in the setting of colitis is important to tumorigenesis.

\section{TNF- $\alpha$ : a link between the immune response and cancer}

The study from Popivanova and colleagues in this issue of the JCI extends this paradigm by examining the role of TNF- $\alpha$ in tumor progression in the AOM/DSS model of CAC (21). TNF- $\alpha$ has previously been implicated in the development of colitis, and indeed pharmacologic blockade of TNF- $\alpha$ with monoclonal antibodies has demonstrated great efficacy in the treatment of IBD patients (22). TNF- $\alpha$ has also been implicated as a positive factor in the development of some other epithelial malignancies, particularly skin cancer in mice (23), raising the possibility that TNF- $\alpha$ may play a similar tumor-promoting role in CAC. In light of the prior data on TNF- $\alpha$, Popivanova et al. examined the specific contribution of TNF- $\alpha$ signaling in CAC, using mice deficient in type I TNF receptor p55 (TNF-Rp55) (21). Akin to what is observed in humans with IBD, abrogation of TNF signaling in mice greatly ameliorated their colitis, as could be gleaned from the decreased tissue injury, inflammatory cell infiltrates, and cytokine expression in the mucosa. Loss of TNF signaling also greatly suppressed CAC. The decreased likelihood of CAC, as a result of TNF receptor deficiency, was recapitulated in wild-type animals by pharmacologic inhibition of TNF- $\alpha$ with etanercept, a recombinant fusion protein resuling from fusion between the Fc portion of human IgG and the ligand-binding region of the TNF type I receptor.

\section{TNF- $\alpha$ activates an oncogenic immune response}

In light of the participation of epithelial cells and inflammatory cells in the tumorigenic process, the identification of the cell population that responds to TNF- $\alpha$ and that promotes tumor for- mation was very important to address. In elegant bone marrow chimeric studies, Popivanova et al. (21) found that the deficiency of TNF-Rp55 was only relevant when it involved immune system cells and not when it involved the epithelium. This indicates that TNF- $\alpha$ production in the mucosa, presumably initiated by resident cells, is required for activation of immune cells, which in turn are important in the subsequent development of cancer (Figure 1). TNF- $\alpha$ did not appear to behave as a trophic factor for the epithelium, but was needed to activate a tumor-promoting immune response. In addition, an intriguing result reported by Popivanova et al., albeit one that lacks clear mechanistic insights at present, was the authors' finding that TNF- $\alpha$ blockade by etanercept may have had inhibitory effects on the outgrowth and/or persistence of neoplastic colon epithelial cells harboring $\beta$-catenin mutations. Mutational defects abrogating regulation of $\beta$-catenin signaling (e.g., adenomatous polyposis coli gene inactivation) have been suggested to be a late event in the pathogenesis of CAC in humans (24). As such, it remains to be determined whether the association that Papivanova et al. observed between TNF blockade and a reduced $\beta$-catenin mutation frequency in neoplastic lesions in the mice reflects a direct stimulatory interaction between TNF signaling and $\beta$-catenin or the fact that loss of TNF signaling inhibited progression of neoplastic cells to the state at which they might acquire $\beta$-catenin dysregulation.

In closing, it is worth emphasizing that the observations reported in the Popivanova et al. (21) study have potentially significant clinical ramifications. While etanercept is approved for clinical use for rheumatoid arthritis, it lacks the efficacy that monoclonal antibodies against TNF have in inducing clinical remission in IBD. Nevertheless, it is likely that monoclonal antibodies against TNF would recapitulate the effect of etanercept in this model. Given the time course for development of CAC in humans, it will be some time before we know whether TNF blockade has a significant effect on inhibiting tumor formation in IBD patients, but the rationale for the potential efficacy of TNF blockade in CAC is now well supported by the findings in the current study.

Address correspondence to: Ezra Burstein or Eric R. Fearon, Molecular Mechanisms of Disease Program, University of Michigan School of Medicine, 109 Zina Pitcher Place, Biomedical Science Research Building, 1st Floor, Ann Arbor, Michigan 48109-2200, USA. Phone: (734) 764-1549; Fax: (734) 647-7950; E-mail: fearon@ umich.edu (E.R. Fearon). Phone: (734) 615-1172; Fax: (734) 647-7950; E-mail: ezrab@umich.edu (E. Burstein).

1. Loftus, E.V., Jr. 2004. Clinical epidemiology of inflammatory bowel disease: incidence, prevalence, and environmental influences. Gastroenterology. 126:1504-1517.

2. Podolsky, D.K. 2002. Inflammatory bowel disease. N. Engl. J. Med. 347:417-429.

3. Itzkowitz, S.H., and Harpaz, N. 2004. Diagnosis and management of dysplasia in patients with inflammatory bowel diseases. Gastroenterology. 126:1634-1648

4. Sands, B.E. 2007. Inflammatory bowel disease: past, present, and future. J. Gastroenterol. 42:16-25.

5. Hampe, J., et al. 2001. Association between insertion mutations in NOD2 gene and Crohn's disease in German and British populations. Lancet. 357:1925-1928

6. Hugot, J.P., et al. 2001. Association of NOD2 leucine-rich repeat variants with susceptibility to Crohn's disease. Nature. 411:599-603.

7. Ogura, Y., et al. 2001. A frameshift mutation in NOD2 associated with susceptibility to Crohn's disease. Nature. 411:603-606.

8. Girardin, S.E., Hugot, J.P., and Sansonetti, P.J. 2003. Lessons from Nod 2 studies: towards a link between Crohn's disease and bacterial sensing. Trends Immunol. 24:652-658.

9. Tanaka, T., et al. 2003. A novel inflammation-related mouse colon carcinogenesis model induced by azoxymethane and dextran sodium sulfate. Cancer Sci. 94:965-973.

10. Andresen, L., et al. 2005. Activation of nuclear factor $\kappa \mathrm{B}$ in colonic mucosa from patients with collagenous and ulcerative colitis. Gut. 54:503-509.

11. Hacker, H., and Karin, M. 2006. Regulation and function of IKK and IKK-related kinases. Sci. STKE. 357:re13.

12. Neurath, M.F., Pettersson, S., Meyer zum Buschenfelde, K.H., and Strober, W. 1996. Local administration of antisense phosphorothioate oligonucleotides to the p65 subunit of NF-KB abrogates established experimental colitis in mice. Nat. Med. 2:998-1004.

13. Neurath, M.F., and Pettersson, S. 1997. Predominant role of NF- $\mathrm{KB}$ p 65 in the pathogenesis of chronic intestinal inflammation. Immunobiology. 198:91-98.

14. Lawrance, I.C., et al. 2003. A murine model of chronic inflammation-induced intestinal fibrosis down-regulated by antisense NF- $\mathrm{B}$. Gastroenterology. 125:1750-1761.

15. MacMaster, J.F., et al. 2003. An inhibitor of IкB kinase, BMS-345541, blocks endothelial cell adhesion molecule expression and reduces the severity of dextran sulfate sodium-induced colitis in mice. Inflamm. Res. 52:508-511.

16. Gilmore, T.D., Kalaitzidis, D., Liang, M.C., and Starczynowski, D.T. 2004. The c-Rel transcription factor and B-cell proliferation: a deal with the devil. Oncogene. 23:2275-2286.

17. Greten, F.R., et al. 2004. IKKb links inflammation and tumorigenesis in a mouse model of colitisassociated cancer. Cell. 118:285-296.

18. Becker, C., et al. 2004. TGF-beta suppresses tumor progression in colon cancer by inhibition of IL- 6 trans-signaling. Immunity. 21:491-501.

19. Courtois, G. 2005. The NF-kappaB signaling path- 
way in human genetic diseases. Cell. Mol. Life Sci. 62:1682-1691.

20. Zhang, J., et al. 2006. Impaired regulation of $\mathrm{NF}-\mathrm{KB}$ and increased susceptibility to colitis-associated tumorigenesis in CYLD-deficient mice. J. Clin. Invest. 116:3042-3049.
21. Popivanova, B.K., et al. 2008. Blocking TNF- $\alpha$ in mice reduces colorectal carcinogenesis associated with chronic colitis. J. Clin. Invest. 118:560-570.

22. Rutgeerts, P., et al. 2005. Infliximab for induction and maintenance therapy for ulcerative colitis. N. Engl.J. Med. 353:2462-2476.
23. Moore, R.J., et al. 1999. Mice deficient in tumor necrosis factor- $\alpha$ are resistant to skin carcinogenesis. Nat. Med. 5:828-831.

24. Itzkowitz, S.H. 2006. Molecular biology of dysplasia and cancer in inflammatory bowel disease. Gastroenterol. Clin. North Am. 35:553-571.

\title{
Erythropoietin: when liability becomes asset in neurovascular repair
}

\author{
Maria B. Grant, ${ }^{1}$ Michael E. Boulton, ${ }^{2}$ and Alexander V. Ljubimov ${ }^{3}$
}

\begin{abstract}
1Program in Stem Cell Biology and Regenerative Medicine, Department of Pharmacology and Therapeutics, University of Florida, Gainesville, Florida, USA. ${ }^{2}$ Age-Related Macular Degeneration Center, Department of Ophthalmology and Visual Sciences, University of Texas Medical Branch, Galveston, Texas, USA. ${ }^{3}$ Ophthalmology Research Laboratories, Cedars-Sinai Medical Center, and UCLA David Geffen School of Medicine, Los Angeles, California, USA.
\end{abstract}

\begin{abstract}
Erythropoietin (Epo) leads to the proliferation and differentiation of erythroid precursors, but is also involved in diverse nonhematopoietic biological functions. In this issue of the JCI, Chen, Smith, and colleagues demonstrate that the temporal expression of Epo is critical for determining whether physiological or pathological repair occurs following neurovascular retinal injury in the oxygen-induced retinopathy neonatal mouse model (see the related article beginning on page 526). The pleiotrophic properties of Epo make it a likely novel therapy for treatment of neurovascular damage, but the timing of its use must be carefully considered to prevent untoward effects.
\end{abstract}

In 1994, Smith and coworkers (1) developed and refined a neonatal mouse model of proliferative oxygen-induced retinopathy (OIR; Figure 1). In this model, 7-dayold mouse pups with partially developed retinal vasculature are subjected to hyperoxia ( $75 \%$ oxygen) for 5 days, which stops retinal vessel growth and causes significant vaso-obliteration. On postnatal day 12 , the pups are returned to room air, and by postnatal day 17 , a florid compensatory retinal neovascularization occurs. This model of pathological neovascularization has been widely used as a substitute for proliferative diabetic retinopathy (DR) and in studies of various antiangiogenic compounds (2-4).

In this issue of the JCI, Chen, Smith, and associates highlight the importance of temporal expression of the hormone erythropoietin (Epo) in the pathogenesis of retinopathy using the OIR model (5).

Nonstandard abbreviations used: DR, diabetic retinopathy; EPC, endothelial progenitor cell; Epo, erythropoietin; EpoR, Epo receptor; OIR, oxygen-induced retinopathy; ROP, retinopathy of prematurity.

Conflict of interest: The authors have declared that no conflict of interest exists.

Citation for this article: J. Clin. Invest. 118:467-470 (2008). doi:10.1172/JCI34643.
They report that during the hyperoxic phase of the OIR model, mRNA levels of retinal Epo were suppressed, while during the subsequent hypoxic phase Epo mRNA levels were greatly increased (Figure 1). Early administration of Epo protected against hyperoxia-induced retinal vaso-obliteration and neural apoptosis, preventing vessel dropout and hypoxiainduced neovascularization in the second phase of this disease model. However, treatment with exogenous Epo during the second phase of the OIR model actually increased pathological neovascularization. Furthermore, Chen et al. (5) also show that hyperoxia resulted in the loss of bone marrow-derived (BM-derived) endothelial progenitor cell (EPC) contribution to vascular development (2). During retinal development, the suppression of endogenous growth factors by hyperoxia with subsequent vaso-obliteration is the critical initiating event responsible for the development of pathological neovascularization. This aberrant neovascularization is responsible for the visionthreatening aspects of retinopathy of prematurity (ROP) and is also seen in DR $(6,7)$. Patients with these conditions are treated once pathological vessels appear.
However, treating "late-stage" disease may be much less effective and, as shown in the current study by Chen et al. (5), early treatment may be a more judicious approach, at least in the case of Epo.

\section{Epo as a vasculogenic, neuroprotective, and EPC recruitment factor}

The recent identification of critical hypoxia-regulated factors and the temporal changes in their expression represents an important step toward understanding mechanisms of disease progression in retinal neovascularization. The pharmaceutical industry has aggressively identified agents to specifically target late events in disease development when neovascularization is already present and to inhibit the hypoxia-regulated factors implicated in pathological neovascularization $(8,9)$. Most of these factors have intrinsic neural and retinal vascular protective effects, and their elimination may eventually contribute to cell and tissue dysfunction. More importantly, their expression represents a physiological adaptive response to ischemic tissue injury.

One such factor, Epo, is a glycoprotein (molecular mass is approximately $30 \mathrm{kDa}$ ) produced primarily in the kidney but with localized production also reported in a range of neural and nonneural tissues (10, 11). Epo is directly activated by the transcription factors HIF-1 $\alpha$ (12), HIF- $1 \alpha-$ like factor (HLF, also known as EPAS1) (13), and HIF-2 $\alpha$ (14) and thus is induced in direct response to ischemic or hypoxic conditions. The Epo receptor (EpoR) also appears to be widely expressed, and ligand binding is associated with activa- 